\title{
CROMATOGRAFÍA LÍQUIDA Y ESPECTROMETRÍA DE MASA EN TÁNDEM, PARA LA DETECCIÓN DE RESIDUOS DE NITROFURANOS EN MÚSCULO DE CERDO EN LA CIUDAD DE TACNA
}

\author{
LIQUID CHROMATOGRAPHY AND THE ASS SPECTROMETRY TANDEM \\ FOR THE DETECTION OF RESIDUES OF NITROFURANS IN MUSCLE \\ PORK IN TACNA"
}

${ }^{1}$ Yoannis Milovna Butrón Solis

\begin{abstract}
RESUMEN
El presente estudio se realizó en el camal Municipal de Tacna, con el objetivo de determinar la presencia de los residuos de nitrofuranos en músculo de cerdo, se tomaron 15 muestras de 500 gramos c/u. Estas muestras en el laboratorio fueron analizadas mediante la técnica de LC/MS-MS los resultados obtenidos fueron el $40 \%$ de las muestras presentaron residuos del metabolito Furaltadona (AMOZ) detectándose desde $0,500 \mu \mathrm{g} / \mathrm{kg}$ hasta $2,059 \mu \mathrm{g} / \mathrm{kg} \mathrm{n}=6$ y el $60 \%$ no presentó ninguno de los otros metabolitos Furazolidona (AOZ) Nitrofurazona (SEM) Nitrofurantoína (ADH) n=9. De manera paralela se hicieron encuestas a porcicultores (15) y a consumidores (150), sobre el uso de antibióticos y sus consecuencias en la salud pública. No tienen asesoramiento técnico el $73 \%$ de los criadores y el $46,7 \%$ tiene poco o nada de conocimientos sobre antibióticos, sin embargo usan nitrofuranos $46,7 \%$ como promotores de crecimiento y $26,7 \%$ para sanidad, no conocen cuánto es el periodo de retiro un $46,7 \%$ y el $53,3 \%$ de los criadores desconocen los problemas sanitarios que causa en el consumidor el no respetar dichos periodos. Consumen carne de cerdo en ocasiones especiales un $53,3 \%$, los consumidores desconocen un $76,7 \%$ sobe los problemas sanitarios que ocasionan el consumo de éstas carnes. En conclusión se considera una carne no apta para el consumo humano porque representa un riesgo a la salud pública y se recomienda que las autoridades e instituciones sanitarias hagan cumplir las normas vigentes además de la capacitación tanto de porcicultores como de consumidores.
\end{abstract}

Palabras clave: LC/MS-MS, metabolitos, nitrofuranos, periodo de retiro, residuos

\begin{abstract}
ABSTRAC
This study was conducted at the Municipal slaughterhouse of Tacna, in order to determine the presence of nitrofuran residues in pork muscle technique LC / MS-MS, 15 samples of 500 grams c / $\mathrm{u}$ is taken, the results were: $40 \%$ of the samples had residues of the metabolite Furaltadone (AMOZ) detected from $0,500 \mu \mathrm{g} / \mathrm{kg}$ to $2,059 \mu \mathrm{g} / \mathrm{kg} \mathrm{n}=6$ and $60 \%$ did not present any of the other metabolites furazolidone (AOZ) nitrofurazone(SEM) nitrofurantoin $(\mathrm{ADH}) \mathrm{n}=9$. In parallel surveys were made to hog producers (15) and consumers (150) on the use of antibiotics and its impact on public health. They have expertise $73 \%$ of farmers and $46,7 \%$ have little or no knowledge about antibiotics, however $46,7 \%$ Nitrofurans used as growth promoters and $26,7 \%$ for health, do not know how much is the withdrawal period by $46,7 \%$ and $53,3 \%$ of the farmers are unaware of the health problems it causes in the consumer does not respect these periods. Pork consumed on special occasions $53,3 \%$, consumers are unaware $76,7 \%$ SoBe health problems caused by the consumption of these meats. Under these conditions it is considered unfit for human consumption meat because it represents a risk to public health and recommends that authorities and health institutions to enforce existing rules plus training both pig farmers and consumers
\end{abstract}

Keywords: LC / MS-MS, metabolites, nitrofurans, withdrawal period, residues

\section{INTRODUCCIÓN}

El uso de medicamentos veterinarios es esencial durante la crianza de animales productores de alimentos, son empleados con fines terapéuticos y preventivos y en otros casos se aplican como promotores de crecimiento. En los últimos años el sector agroalimentario en todo el mundo se ha enfrentado a la diseminación de brotes de enfermedades transmitidas por alimentos en los que intervienen, entre otros agentes, residuos de medicamentos veterinarios de origen animal, que generan productos de baja calidad y constituyen un riesgo para la salud de los consumidores, produ-

'Egresada de Medicina Veterinaria y Zootecnia, Laboratorio de Microbiología, Facultad de Ciencias Agropecuarias, Universidad Nacional Jorge Basadre Grohmann. 
Butrón, Y. Cromatografia líquida y espectrometría de masa en tándem, para la detección de residuos de nitrofuranos en músculo de cerdo en Tacna

ciendo toxicidad aguda y crónica, efectos mutagénicos y carcinógenos, desórdenes en el desarrollo corporal, reacciones alérgicas y resistencia bacteriana, entre otros. Estos efectos adversos han hecho que organizaciones internacionales regulen con fundamento científico los residuos de fármacos de uso veterinario potencialmente peligrosos para la salud (Lozano \& Arias, 2008).

A pesar de la antigüedad de las regulaciones internacionales existentes, solo hasta hace poco en Perú y en la región de Tacna, dada la actual situación económica y comercial de esta problemática sanitaria y se han comenzado a adoptar nuevas medidas para reconocer la residualidad de fármacos y de otras sustancias en los alimentos de origen animal producidos en el país, el Perú con su organismo regulador el Servicio Nacional de Sanidad y Calidad Agroalimentaria (SENASA) aún no cuenta con una reglamentación específica establecida para los cuatro metabolitos, la cual pueda regir la salubridad pública con respecto a este tema.

El objetivo fue detectar la presencia de residuos de nitrofuranos y/o de sus metabolitos en el músculo de cerdo beneficiados en el Camal Municipal Eduardo Eyzaguire Yañez;

\section{MATERIALES Y MÉTODOS}

\section{Materiales}

Para la investigación del presente estudio se tomaron 15 muestras de músculo de cerdo, deshuesado y libres de grasa $(500 \mathrm{~g})$, las cuales fueron remitidas a laboratorio de Centro de Control de Insumos y Residuos Tóxicos de SENASA - LIMA, mediante el método de Cromatografía Líquida y Espectrometría de Masa en Tándem (LC/MSMS). El muestreo, su procesamiento y análisis se realizó en los meses de Octubre del 2014 a Mayo 2015.

\section{Metodología}

La investigación se realizó con un modelo descriptivo transversal. Para determinar el tamaño de la población, se usó como referencia el número diario de cerdos beneficiados en el camal Municipal, para el tamaño de muestra se utilizó la fórmula para variables cualitativas de tipo finito y en base a ello se obtuvo un número de 15 muestras. De manera paralela se realizaron dos encuestas a los porcicultores 15 (siendo el mismo número que las muestras por tener la misma confiabilidad estadística). Y otra a los consumidores 150 de los diferentes distritos de la ciudad.

Para las encuestas a los consumidores se tomó como referencia al número de familias proyectadas por el INEI para el 2012. El tamaño de la muestra a un nivel de confianza de $95 \%$ y con un margen de error del $8 \%$ fue de 150 encuestas, luego consideramos como estratos a cada uno de los distritos que conforman la urbanización de la ciudad de Tacna, son los Distritos de: Tacna (53), Alto de la Alianza (20), Ciudad Nueva (19), Gregorio Albarracín (48), y finalmente Pocollay con (10) encuestas.

\section{RESULTADOS Y DISCUSIÓN}

En la tabla 1 se observa que se ha detectado el metabolito Furaltadona (AMOZ) en las muestras: 1,2,7,8,9 y 10 con límites de detección que van desde $0,500-2,059 \mu \mathrm{g} / \mathrm{kg}$ de carne en músculo de cerdo, en las muestras restantes $3,4,5,6,11,12,13,14$ y 15 no se detectaron los metabolitos AOZ, SEM y ADH.

Todos las muestras remitidas al Laboratorio de SENASA en Lima se analizaron con la técnica de Cromatografía Líquida y espectrometría de Masas en Tándem (LCMS/MS), siendo ésta sensible, confiable y confirmatoria, para identificar y cuantificar los cuatro metabolitos de nitrofuranos (Leitner et al., 2001). La LC-MS/MS es diez veces más sensible que la cromatografía líquida con detección ultravioleta (HPLC-UV) en la detección de Furazolidona y furaltadona, es dos veces más sensible para nitrofurazona, mientras LC-MS/MS y HPLC-UV son igualmente sensibles para la detección de nitrofurantoína (Cooper et al., 2005). Según Xia et al. (2008) la técnica LC-MS/MS demostró ser

Tabla 1. Resumen de resultados de muestras remitidas al laboratorio de SENASA - LIMA

\begin{tabular}{|c|c|c|c|c|}
\hline \multirow[b]{2}{*}{$\mathrm{N}^{\circ}$ de Muestras } & \multicolumn{4}{|c|}{ Residuos de Metabolitos de nitrofuranos (ug/kg) } \\
\hline & $\begin{array}{c}\text { Furazolidona } \\
\text { 3-amino-oxazolidinona } \\
\text { (AOZ) }\end{array}$ & $\begin{array}{l}\text { Furaltadona } \\
\text { 3-amino-5-morpholinomethyl-1,3-oxazolidina } \\
\text { (AMOZ) }\end{array}$ & $\begin{array}{l}\text { Nitrofurantoina } \\
\text { 1-aminohydantoina } \\
\text { (AHD) }\end{array}$ & $\begin{array}{l}\text { Nitrofurazona } \\
\text { Semicarbazida } \\
\text { (SEM) }\end{array}$ \\
\hline 1 & N.D. & 1,823 & N.D. & N.D. \\
\hline 2 & N.D. & 0,817 & N.D. & N.D. \\
\hline 3 & N.D. & N.D. & N.D. & N.D. \\
\hline 4 & N.D. & N.D. & N.D. & N.D. \\
\hline 5 & N.D. & N.D. & N.D. & N.D. \\
\hline 6 & N.D. & N.D. & N.D. & N.D. \\
\hline 7 & N.D. & 0,500 & N.D. & N.D. \\
\hline 8 & N.D. & 2,059 & N.D. & N.D. \\
\hline 9 & N.D. & 0,763 & N.D. & N.D. \\
\hline 10 & N.D. & 0,870 & N.D. & N.D. \\
\hline 11 & N.D. & N.D. & N.D. & N.D. \\
\hline 12 & N.D. & N.D. & N.D. & N.D. \\
\hline 13 & N.D. & N.D. & N.D. & N.D. \\
\hline 14 & N.D. & N.D. & N.D. & N.D. \\
\hline 15 & N.D. & N.D. & N.D. & N.D. \\
\hline
\end{tabular}

ND: No detectable

Fuente: Laboratorio de Insumos y Residuos Tóxicos, SENASA-LIMA 
Butrón, Y. Cromatografía líquida y espectrometría de masa en tándem, para la detección de residuos de nitrofuranos en músculo de cerdo en Tacna

un procedimiento preciso, simple, sensible y fiable para el análisis y confirmación de nitrofuranos en carne de cerdo y, Jun Li et al. (2009) indican que ELISA puede ser una herramienta práctica para la detección de los cuatro nitrofuranos en alimentos para animales, sin embargo luego debe de usarse la técnica de Cromatografía Líquida para la confirmación.

En la tabla 2 y figura 1 se observa que 6 de las 15 muestras de músculo de cerdo analizadas contienen residuos de Furaltadona (AMOZ), las 9 restantes no contienen ningún tipo de residuos de nitrofuranos.

Tabla 2. Presencia de residuos de metabolitos de nitrofuranos en músculo de cerdo-Tacna

\begin{tabular}{lll}
\hline Nitrofuranos & Frecuencia & Porcentaje \\
\hline Ninguno & 9 & 60,0 \\
Furaltadona (AMOZ) & 6 & 40,0 \\
Total & 15 & 100,0 \\
\hline
\end{tabular}

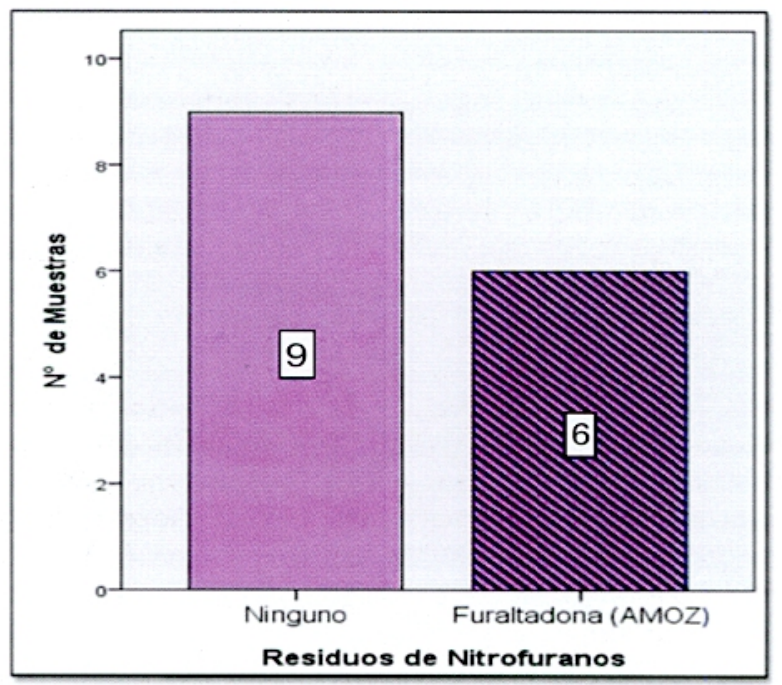

Figura 1. Residuos de metabolitos de nitrofuranos en músculo de cerdo-Tacna

Se concluye que en $40 \%$ de las muestras de músculo de cerdo presentan residuos de al menos de un metabolito (Furaltadona-AMOZ) y el $60 \%$ no contiene ningún residuos de nitrofurano.

Según la figura 1 la presencia del metabolito AMOZ es altamente significativa con un $95 \%$ de confianza estadística denotando así que la carne que se genera en el camal no es apta para el consumo humano.

Los resultados del presente trabajo son diferentes a los obtenidos por SENASA-LIMA en el 2012 (SENASA, 2014) ya que ellos reportan un $100 \%$ en las tres muestras enviadas de los distritos de Tacna para el metabolito furazolidona (AOZ), y con niveles menores a $1 \mu \mathrm{g}$; esto se debe al tamaño de muestra, ya que en la presente investigación se analizaron 15 muestras y en el caso de ellos, solamente fueron tres. Por otro lado en el presente trabajo se ha detectado el metabolito furaltadona (AMOZ), en cambio, SENASA (2012) detecta AOZ, esto se debería a que los productores de porcinos están usando ambos nitrofuranos. Los niveles de furaltadona hallado en nuestro trabajo van desde 0,500 $2,059 \mu \mathrm{g} / \mathrm{kg}$ de carne, y que son superiores a lo reportado por SENASA LIMA en el 2012 (SENASA, 2014), quienes obtuvieron niveles menores a $1 \mu \mathrm{g} / \mathrm{kg}$, esta diferencia podría deberse a que las muestras utilizadas en el presente trabajo, podrían ser de animales, que estuvieron en tratamiento con el Nitrofurano Furaltadona y en el caso de las muestras de SENASA podrían ser de animales que sòlo han estado recibiendo el producto como promotores de crecimiento. Esto indica que hay incumplimiento de la R. D.N $0072-2013$ MINAGRI - SENASA - DIAIA donde menciona la prohibición y comercialización de productos que contengan nitrofuranos como principio activo y del Reglamento de la UE N³7/2010, y el Anexo IV del Reglamento $N^{\circ}$ 2377/90 que indica Tolerancia Cero para nitrofuranos por su potencial mutagénico por lo que no se puede establecer LMR en carne para consumo humano y que según el comité mixto OMS/FAO establecen a los nitrofuranos desde 1995 como medicamentos prohibidos.

Estos resultados también son inferiores a los obtenidos por SENASA LIMA en el año 2013-2014 quienes analizaron 7 muestras de los cuales el $57 \%$ fueron positivos a furazolidona (AOZ), esto también se debe a que los porcicultores usan tanto furazolidona o furaltadona; durante el periodo en que se han tomado las muestras probablemente éste antibiótico no se ha estado usando en la crianza de cerdos, y que el muestreo se haya realizado entre la primavera y verano, meses en los cuales el clima no favorece enfermedades digestivas pero si respiratorias esto concuerda con las encuestas en donde las estas enfermedades respiratorias son más comunes en las granjas y se utiliza el nitrofurano AMOZ en su tratamiento; en cuanto a los niveles encontrados por SENASA en el 2013-2014 son bastante superiores ya que ellos, detectan niveles desde 12 a $22 \mu \mathrm{g} / \mathrm{kg}$ de carne de cerdo, y esto podría deberse a que las muestras analizadas provienen de animales que han estado en tratamiento.

Tal como ha sido reportado por Cooper et al. (2005), quienes utilizando una dosis terapéutica de $400 \mathrm{mg} / \mathrm{kg}$ del nitrofurano furazolidona en porcinos encuentran que, aún después de 6 semanas del cese del tratamiento, la vida media del metabolito AOZ es mayor en el músculo de cerdo y más corta en el riñón, en tiempo de espera cero se detectó $(330 \mu \mathrm{g} / \mathrm{kg})$ utilizando la técnica LC-MS / MS y utilizando la misma técnica Lucas \& Olivos (2007) reportan haber encontrado $0,07 \mathrm{ng} / \mathrm{g}$ del metabolito AOZ en músculo de pollo.

Los resultados son similares a los reportes de Barrios (2011) quien encontró que el $90 \%$ de las muestras de músculo y $60 \%$ de las muestras de hígado de pollo presentan niveles de residuos de nitrofuranos menores a $0,0001 \mu \mathrm{g} / \mathrm{kg}$ de músculo $\mathrm{y} / \mathrm{o}$ hígado; mientras que un menor porcentaje presentan niveles de residuos ligeramente superior a $0,0001 \mu \mathrm{g} / \mathrm{kg}$ de nitrofuranos lo cual implica que se sigue usando este antibiótico como promotor de crecimiento en aves.

\section{CONCLUSIONES}

Las muestras de músculo de cerdo beneficiadas en la ciudad de Tacna analizadas mediante la técnica LC-MS-MS, 
Butrón, Y. Cromatografía líquida y espectrometria de masa en tándem, para la detección de residuos de nitrofuranos en músculo de cerdo en Tacna

no presentan residuos de los metabolitos AOZ, SEM y $\mathrm{ADH}$; pero sí se detecta el metabolito AMOZ del nitrofurano furaltadona (FTD), considerándose así una carne no apta para el consumo humano porque representa un riesgo para la salud pública.

Los porcicultores desconocen sobre el uso de antibióticos nitrofuranos, sin embargo medican sin prescripción médica a sus animales y no toman en cuenta las consecuencias que ocasionan a los consumidores.

La mayor población de Tacna que consume carne de cerdo desconoce los problemas sanitarios que provocan los porcicultores al no respetar el periodo de retiro de los antibióticos en la crianza de porcinos.

\section{AGRADECIMIENTOS}

A la Universidad Nacional Jorge Basadre Grohmann que a través del concurso de financiamiento de tesis con los fondos de canon, sobrecanon y regalías mineras, apoyo el desarrollo de esta investigaciòn. Al MSc L. Barrios Moquillaza por la revisión del manuscrito y al MSc. E. Palza por los análisis estadísticos.

\section{REFERENCIAS BIBLIOGRÁFICAS}

Barrios, L. A. (2011). Estudio de los niveles de residuos de antibióticos en musculo e hígado de pollos beneficiados en la ciudad de Tacna (Tesis de Maestría). Universidad Nacional Jorge Basadre Grohmann, Tacna, Perú

Cooper, K. M., Mulder, P. P. J., Van Rhijn, J. A, Kovacsics, L., McCracken, R. J., Young, P. B. \& Kennedy, D. G. (2005). Depletion of four nitrofuran antibiotics and their tissue-bound metabolites in porcine tissues and determination using LC-MS/MS and HPLC-UV.
Food Additives and Contaminants, 22(5), 406-414. http://doi.org/10.1080/02652030512331385218

Leitner, A., Zöllner, P. \& Lindner, W. (2001). Determination of the metabolites of nitrofuran antibiotics in animal tissue by high-performance liquid chromatography - Tandem mass spectrometry. Journal of Chromatograpby A, 939(1-2), 49-58. http://doi.org/10.1016/S0021$9673(01) 01331-0$

Li, J., Liu, J. X. \& Wang, J. P. (2009). Multidetermination of four nitrofurans in animal feeds by a sensitive and simple enzyme-linked immunosorbent assay. Journal of Agricultural and Food Chemistry, 57(6), 2181-2185. http://doi.org/10.1021/jf8035098

Lozano, M. \& Arias, D. (2008). Residuos de fármacos en alimentos de origen animal: panorama actual en Colombia. Colombian Journal of Animal Science and Veterinary Medicine, 21(1). Recuperado desde http://www.scielo.org.co/scielo.php?script=sci_ser ial\&lng=en\&pid $=0034-7450 \& \mathrm{nrm}=$ iso

Lucas, O. \& Olivos, H. (2007). Cromatografía líquida acoplada a espectrometría de masa cuadrupolar en tándem (LC / MS / MS) y su aplicación en el análisis de residuos de antibióticos nitrofuranos en músculo de pollo. Control, 21-28. Recuperado desde http://departamento.pucp.edu.pe/ciencias/images /documentos/XXI-Orlando_Lucas.pdf

SENASA. (2014). Monitoreo Senasa 2012- 2013. Tacna, Perú.

Xia, X., Li, X., Zhang, S., Ding, S., Jiang, H., Li, J. \& Shen, J. (2008). Simultaneous determination of 5nitroimidazoles and nitrofurans in pork by highperformance liquid chromatography-tandem mass spectrometry. Journal of Chromatography A, 1208(1-2), 101-108. http://doi.org/10.1016/j.chroma.2008.08.055

\begin{tabular}{ll}
\hline Correspondencia: & Fecha de Recepción: 15/09/2015 \\
Yoannis Milovna Butrón Solis: yoannis.milovna@hotmail.com & Fecha de Aceptación: 02/12/2015
\end{tabular}

